\section{P42 LIFECOURSE CONSEQUENCES OF PARENT TRAINING: MICROSIMULATION MODELLING OF AN ENGLISH BIRTH COHORT}

I Skarda*, M Asaria, R Cookson. Centre for Health Economics, University of York, York, UK

\subsection{6/jech-2018-SSMabstracts.168}

Background Many public policies have potentially important but poorly understood long-run consequences for health, income, public cost and inequality over the lifecourse. We aim to improve understanding by (i) developing a novel discrete time lifecourse microsimulation model of an English birth cohort, and (ii) using it to extrapolate the lifecourse consequences of a training programme ('Incredible Years') for parents of young children exhibiting antisocial behaviour.

Methods Model: We simulate a cohort of 100,000 English children born in 2000-1, using data from the Millennium Cohort Study (MCS) to describe their characteristics and family circumstances. We model the year-by-year evolution of lifecourse outcomes using difference equations parameterised using quasi-experimental evidence and calibrated against longitudinal survey data. Difference equations vary across four key life stages $(0-4,5-24,25-69$ and $70+)$ to represent the causal pathways linking family circumstances, cognitive and socio-behavioural skills formation, conduct disorder and educational attainment in childhood to diverse later life outcomes including poverty, imprisonment, social security benefits, residential care, unhealthy behaviour, physical illness, mental illness and mortality.

Intervention: We assume training is offered to parents of all children at high-risk of conduct disorder at age 5. Effects on the parent-reported Strengths and Difficulties Questionnaire Conduct Subscale are drawn from a participant-level metaanalysis, with effect size differentiated by child gender, baseline behaviour problems and parental mental health.

Primary outcomes: We summarise lifetime benefits using 'good life-years' which go beyond conventional qualityadjusted life-years to adjust for income as well as illness. We examine differential benefits by parental income, mental health and baseline behavioural problems.

Results We estimate that parent training increases the lifetime wellbeing of a child at risk of conduct disorder by an average of 2.43 [95\% CI 1.03 to 3.83] good years. On average, children with parents suffering mental health problems gain 8.04 [95\% CI 6.75 to 9.34] good years; children with severe baseline behavioural problems gain 5.37 [95\% CI 3.97 to 6.77] good years; children that are poorest $20 \%$ at birth gain on average 3.58 [95\% CI 2.63 to 4.54 ] years and children that are richest $20 \%$ at birth gain on average 1.73 [95\% CI 0.77 to 2.69] years. Conclusion Parent training can yield substantial long-run benefits in years of good life gained, especially for children from disadvantaged families, though uncertainty remains about effectiveness for parents who do not seek help.

\section{P43 USING THE HELPMEDOIT! APP AND WEBSITE TO SET AND MONITOR WEIGHT LOSS GOALS AND MOBILISE SOCIAL SUPPORT: QUALITATIVE FINDINGS}

\footnotetext{
1,2 L Matthews* ${ }^{*}$ 1,2 J Pugmire, 1,2S Simpson, 1,2 L Moore. ${ }^{1}$ MRC/CSO Social and Public Health Sciences Unit, Institute of Health and Wellbeing, University of Glasgow, Glasgow, UK; ${ }^{2}$ on behalf of the HelpMeDolt! Trial Management Group
}

10.1136/jech-2018-SSMabstracts.169
Background HelpMeDoIt! is an app/website that harnesses social support for weight loss by enabling participants to nominate friends/family to help them with their weight loss goals. The intervention focused on goal setting, self-monitoring and social support, guided by elements of control theory, social cognitive theory, self-determination theory and social support theories. Objective: To explore the views and experiences of trial participants and their helpers and explore whether the intervention theory was supported.

Methods Qualitative interviews were conducted at $6 \mathrm{mth}$ follow-up, via telephone, with intervention participants $(n=22)$ and their helpers $(\mathrm{n}=9)$. The sample included individuals who had and had not engaged regularly with the app/website. Interviews were recorded, transcribed verbatim and analysed thematically.

Results Insights were gained on various aspects of the intervention, including: feasibility of using an app to set and monitor weight loss goals; the social support process; barriers to engagement; and suggested improvements for a future trial. Key findings indicated that participants and helpers engaged with the concept of the intervention but their participation was hindered to some extent by technical problems. Helpers often provided social support without using the app, e.g. faceto-face or by text. Findings supported several aspects of the intervention theory including: helpers providing instrumental, emotional and informational social support; increased knowledge and skills, sometimes via support from helpers e.g. cooking skills; increased action planning and problem solving via effective goal-setting; and increased motivation either via successful self-monitoring or goal accomplishment. Participant/ helper insight provided several suggestions to enhance the intervention, including, providing a community of peer support.

Conclusion Participants were enthusiastic about having helpers to support them as well as using an app/website for lifestyle behaviour change. The technology needs to be free of technical issues to avoid unnecessary barriers to engagement. An effective weight loss intervention delivered via smartphone app/website has the potential for population-wide impact. Findings will be used to help inform a future full trial.

\section{P44 ABSTRACT WITHDRAWN}

\section{P45 CHILD NUTRIENT INTAKES FROM CURRENT NATIONAL DIETARY SURVEYS OF EUROPEAN POPULATIONS COMPARED TO WHO RECOMMENDATIONS}

${ }^{1}$ HL Rippin*, 'J Hutchinson, ${ }^{2,3}$ J Jewell, ${ }^{2,3} 3 \mathrm{~J}$ Breda, 'JE Cade. 'Nutritional Epidemiology Group, University of Leeds, Leeds, UK; ${ }^{2}$ Division of Noncommunicable Diseases and Promoting Health through the Life-Course, World Health Organization Regional Office for Europe, Copenhagen, Denmark; ${ }^{3}$ World Health Organisation Regional Office for Europe

\subsection{6/jech-2018-SSMabstracts. 170}

Background The World Health Organisation (WHO) encourages national diet survey (NDS) implementation to obtain relevant data to inform policies aimed at addressing the burden of malnutrition and childhood obesity, which remain pressing health issues. However, we have previously shown that European NDS provision is inconsistent, and the majority of gaps lie in Central \& Eastern European countries (CEEC). This paper provides an up-to-date review on energy, macro and 